\title{
The Dynamic Fault Tree Analysis Method Based on Fuzzy Markov Process and Its Application
}

\author{
Xin Liu ${ }^{1}$, Yunxian Jia ${ }^{1}$, Jie Zhou ${ }^{1}$, Zewen He ${ }^{1}$, Yingbo Zhang ${ }^{2}$ \\ 1. Department of Equipment Command and Management, Ordnance Engineering College, \\ Shijiazhuang 050003, China; \\ 2. Department of Aviation Theory Training, Xi'an Flight Academy of Air Force, Xi'an 710306, China \\ Imh19901228@126.com
}

Keywords: Markov process, dynamic fault tree, the triangular fuzzy numbers, reliability

\begin{abstract}
Traditional fault tree analysis is an effective method to analyze system reliability that is based on static logic, and the system which considering the influence in combination of fuzzy uncertainty and dynamic failure characters is still difficult to estimate precisely. The fuzzy number theory can fully consider the fuzzy uncertainty of system which use various aspects information, and Markov process can describe the dynamic failure characteristics of the system through the change of state space, then the fuzzy number theory and Markov process is introduced into dynamic fault tree analysis to overcome the disadvantage of traditional fault tree. The method was applied to dynamic fault tree research of an internal combustion engine to prove the validity.
\end{abstract}

\section{Introduction}

With the development of science and technology, the system of industrial production is becoming more and more complex, and the reliability of complex system has become the key factor to restrict the development of the system ${ }^{[1]}$. Fault Tree Analysis (FTA) is a common method to evaluate the reliability and security of complex systems. With the method of fault tree analysis, we can go to the system of reliability estimation and fault diagnosis and analysis of the weak links of the system, then guide the complex system operation and maintenance activities to achieve system optimization design ultimately ${ }^{[2]}$, as a result, the method is widely used in the field of aerospace, machinery, transportation and ship engineering.

In the traditional fault tree analysis method, the fault of the system and its components are described by the exact probability value, and each unit has two states of normal or fault. In practical application, it is difficult to directly determine the fault probability of each unit by historic statistic data, even, we need to deal with new units which without the fault data, so it is difficult to get accurate fault tree analysis results, as a result, this fault tree analysis methods based on static logic is not suitable for analysis of dynamic and stochastic complex system ${ }^{[3]}$. At the same time, when using the Markov process to deal with the problem, the state space of the model will increase with the size of the complex system, and make the solution of the model become very difficult to solve.

Dynamic fault tree analysis (DFTA) can be the modular model, and the dynamic subtree and the static subtree were established, then we can comprehensive utilization of the advantages of fault tree and Markov process, and simplify the calculation process, which will solve the difficult problems of ask for results when the fault tree turn into a Markov process. Fuzzy mathematics theory can effectively depict less than accurate fault probability of bottom event, it uses fuzzy numbers to describe the probability of occurrence of an event, using fuzzy probability to describe the probability of failure of each unit, and the theory considers the bottom events of the history statistical information, subjective judgment of experts and the random factors into account, can effectively improve the accuracy of estimation of fault tree. In this paper the fuzzy number theory and Markov process are introduced to analyze the dynamic fault tree, and triangular fuzzy numbers are used to describe the failure probability of an event, then the dynamic fault tree fuzzy with Markov process is established, 
finally, the reliability curve of system under the given membership degree is obtainted. Finally, the method is applied to the dynamic fault tree analysis of an internal combustion engine, and the proposed theory is verified.

\section{Application of fuzzy set theory in fault tree analysis}

Fuzzy set theory is used to deal with the problem which is imprecise and fuzzy. The fault events in fault tree can be divided into one which with statistical data or without statistical data and fuzzy event, the fault events with statistical data can obtain the failure rate through access to relevant information, then for the events without statistical data and fuzzy events, the fuzzy set theory can be better to describe the fault rate ${ }^{[5]}$.

In fuzzy set theory, it is assumed that $U$ is a domain of object composition, then a mapping is defined:

$$
\begin{aligned}
\mu_{A}: U & \rightarrow[0,1] \\
u & \rightarrow \mu_{A}(u)
\end{aligned}
$$

$\mu_{A}(u)$ means the membership degree of the fuzzy sets for the elements in the domain which is called the membership degree.

Through fuzzy numbers to analysis the failure probability of fault tree top event, which can make full use of statistical information, but also can effectively deal with all kinds of fuzzy information then we can get more accurate results about reliability analysis and modeling study of complex system. Aiming at the failure probability of bottom event, fuzzy numbers with triangular fuzzy number and trapezoid fuzzy number, normal fuzzy number are commonly used ${ }^{[6]}$, in this paper we use triangular fuzzy number for fault tree analysis, it is assumed that the failure rate of bottom event are triangular fuzzy in order to calculate the failure probability of a logic gate. The membership functions of typical triangular fuzzy numbers are defined as follows:

$$
\mu_{A}= \begin{cases}\frac{x-a}{b-a}, & a \leq x \leq b \\ 1, & \mathrm{x}=\mathrm{b} \\ \frac{x-c}{b-c}, & b<x \leq c \\ 0, & \text { others }\end{cases}
$$

\section{The solution of fuzzy fault tree based on Markov process}

3.1 The Markov process. The basic concepts of the Markov process include the "state" of the system and the "state transfer". The state of system includes work, out of work or part of the failure, and the state transfer of the system means that the state of the system is changed from the fault state to the work state or the other. The basic assumption of the Markov process is that the system state is related to the current state of the system and is not related to its previous history. The mathematical description is that: set $\{X(t), t \geq 0\}$ to a random process in the value of the state space $E=\{0,1, \cdots\}$ or $E=\{0,1, \cdots N\}$, if we set $\mathrm{n}$ time points $0 \leq t_{1}<t_{2} \cdots<t_{n}$, are satisfied

$$
P\left\{X\left(t_{n}\right)=i_{n} \mid X\left(t_{1}\right)=i_{1}, \cdots X\left(t_{n-1}\right)=i_{n-1}\right\}=P\left\{X\left(t_{n}\right)=i_{n} \mid X\left(t_{n-1}\right)=i_{n-1}\right\}
$$

Then $\{X(t), t \geq 0\}$ is the continuous Markov process state in space $\mathrm{E}$.

In the dynamic fault tree analysis, the input event state combination of dynamic logic gate is the basic state of the Markov model, and the transition probability of Markov model state is the fault probability of input event, so that we can convert the dynamic logic gate as a Markov model. 
3.2 Markov algorithm for dynamic fault tree. With the Markov model features of state transition between the various states, solving the dynamic fault tree transformation for solving Markov process can be greatly simplified the calculation process of complex fault tree. Make the fault rate of one or several bottom events of the dynamic fault tree which can change the state of the system as the transition probability of the Markov process and system corresponding to the intermediate state as the intermediate state of the Markov process, then we can construct the corresponding Markov model ${ }^{[7]}$. On the basis of this model, the dynamic fault tree model is solved combined with the triangular fuzzy number theory, and the reliability analysis and modeling of complex system is realized.

In the method, we establish the dynamic fault tree model of the system firstly and dynamic fault tree with N status is transformed by Markov model, then fuzzy set theory is combined. Finally, a Markov process of fuzzy state transition matrix is shown in equation 1.

$$
A=\left(\lambda_{i, j}\right)=\left[\begin{array}{cccc}
\lambda_{1,1} & \lambda_{1,2} & \cdots & \lambda_{1, n} \\
\lambda_{2,1} & \lambda_{2,2} & \cdots & \lambda_{2, n} \\
\vdots & \vdots & \cdots & \vdots \\
\lambda_{n, 1} & \lambda_{n, 2} & \cdots & \lambda_{n, n}
\end{array}\right]
$$

Fig 1 represents the state transition process, where the $S_{0}$ indicates the system that is in good condition; $\mathrm{S}_{\mathrm{i}}(\mathrm{i}=2,3, \ldots \mathrm{N}-1)$ indicates the state that the fault has happened but the system that still can work as a whole, and $S_{n}$ is the fault state of the system.

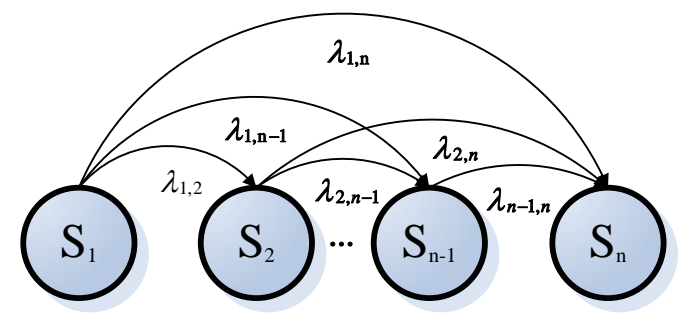

Figure-1 Fuzzy state transfer of the system

The differential equations corresponding to the Markov model is:

$$
\left\{\begin{array}{l}
\frac{d p_{1}(t)}{d t}=-p_{1}(t) \sum_{j=2}^{n} \lambda_{1, j} \\
\frac{d p_{i}(t)}{d t}=\sum_{j=1}^{i-1} p_{j}(t) \lambda_{j . i}-\sum_{j=i+1}^{n} p_{i}(t) \lambda_{i, j}, 1<i<n, t \geq 0 \\
\frac{d p_{n}(t)}{d t}=\sum_{j=1}^{n-1} p_{j}(t) \lambda_{j, n}
\end{array}\right.
$$

Put the initial conditions $p_{1}(0)=1$ and $\quad p_{i}(0)=0(i \neq 1)$ into the equations and obtain the corresponding linear equation with Laplace transformation

$$
\left\{\begin{array}{l}
s p_{1}(s)-1=-p_{1}(s) \sum_{j=2}^{n} \lambda_{1, j} \\
s p_{i}(s)=\sum_{j=1}^{i-1} p_{j}(s) \lambda_{j . i}-\sum_{j=i+1}^{n} p_{i}(s) \lambda_{i, j}, 1<i<n \\
s p_{n}(s)=\sum_{j=1}^{n-1} p_{j}(s) \lambda_{j, n}
\end{array}\right.
$$

The linear equations are solved and the Laplace inverse transform is carried out. Finally, the fuzzy failure probability of the system is obtained. 


\section{Case analysis}

The internal combustion engine has a very wide range of applications, such as automobiles, ships, aircrafts and so on. Based on this, we take a certain internal combustion engine as the research object, and the reliability analysis of it is carried out by the fault tree to verify the method proposed in this paper. Dynamic fault tree of the internal combustion engine is shown in fig 2.

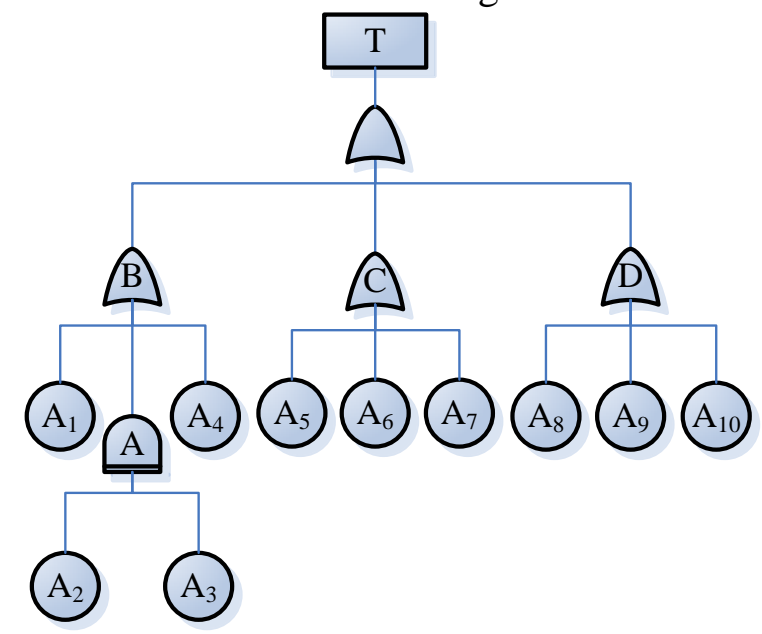

Figure-2 The dynamic fault tree of an internal combustion engine

As seen in Fig 2, the dynamic fault tree bottom event of engine as shown in Table-1, but the probability of bottom event is difficult to obtain accurate values, then based on the engineering experience and expert evaluation, the bottom event probability is represented by fuzzy number method. In table-1, the probability of the occurrence of the bottom events is evaluated by triangular fuzzy numbers.

\begin{tabular}{ccc}
\hline Table-1 the occurrence probability of bottom event \\
\hline & Event & $\begin{array}{c}\text { occurrence } \\
\text { probability }\end{array}$ \\
\hline $\mathrm{A}_{1}$ & carburetor fault & $(0,0.005,0.01)$ \\
$\mathrm{A}_{2}$ & $\begin{array}{c}\text { did not check } \\
\text { the fuel tank }\end{array}$ & $(0,0.02,0.04)$ \\
& oil exhaustion & $(0.06,0.08,0.1)$ \\
$\mathrm{A}_{3}$ & tubing plug & 0.01 \\
$\mathrm{~A}_{4}$ & seal leakage & 0.001 \\
$\mathrm{~A}_{5}$ & piston stuck & 0.0042 \\
$\mathrm{~A}_{6}$ & piston ring fault & 0.001 \\
$\mathrm{~A}_{7}$ & spark plug & $(0.05,0.1,0.15)$ \\
$\mathrm{A}_{8}$ & failure & 0.01 \\
$\mathrm{~A}_{9}$ & line fault & 0.01 \\
$\mathrm{~A}_{10}$ & motor fault & 0 \\
\hline
\end{tabular}

The dynamic fault tree model is transformed into fuzzy Markov model, which $\mathrm{S}_{0}$ represents internal combustion engine in a normal state; $S_{1}$ represents the fault state of part system caused by the internal combustion engine fuel tank uncheck; $S_{2}$ represents the state of the internal combustion engine oil exhaustion; $S_{\mathrm{f}}$ represents the internal combustion engine fault condition. The state transfer process of an internal combustion engine is shown in fig 3. 


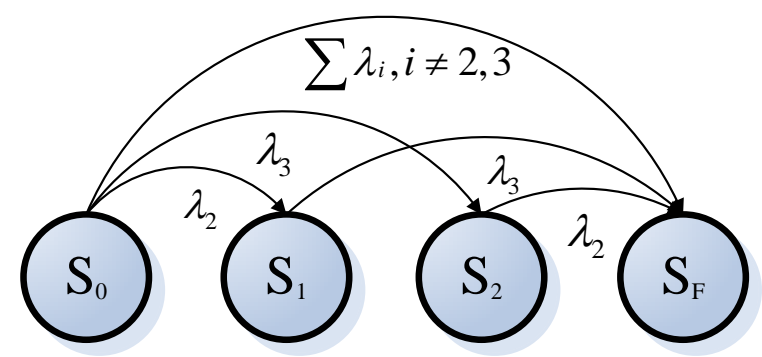

Figure-3 The state transfer of internal combustion engine

The fuzzy Markov transfer matrix of the internal combustion engine is obtained by the state transition diagram:

$$
A=\left[\begin{array}{cccc}
-\sum_{i=1}^{10} \lambda_{i} & \lambda_{2} & \lambda_{3} & \sum_{i=1}^{10} \lambda_{i}(i \neq 2,3) \\
0 & -\lambda_{3} & 0 & \lambda_{3} \\
0 & 0 & -\lambda_{2} & \lambda_{2} \\
0 & 0 & 0 & 0
\end{array}\right]
$$

After getting the matrix, the fuzzy failure probability function of internal combustion engine failure can be obtained by the formula 2 and formula 3. The results are shown in figure-4. As we can see from fig 4 , the value of the fuzzy probability is about 0.19 , which indicates that the maximum probability of internal combustion engine failure is 0.19 .

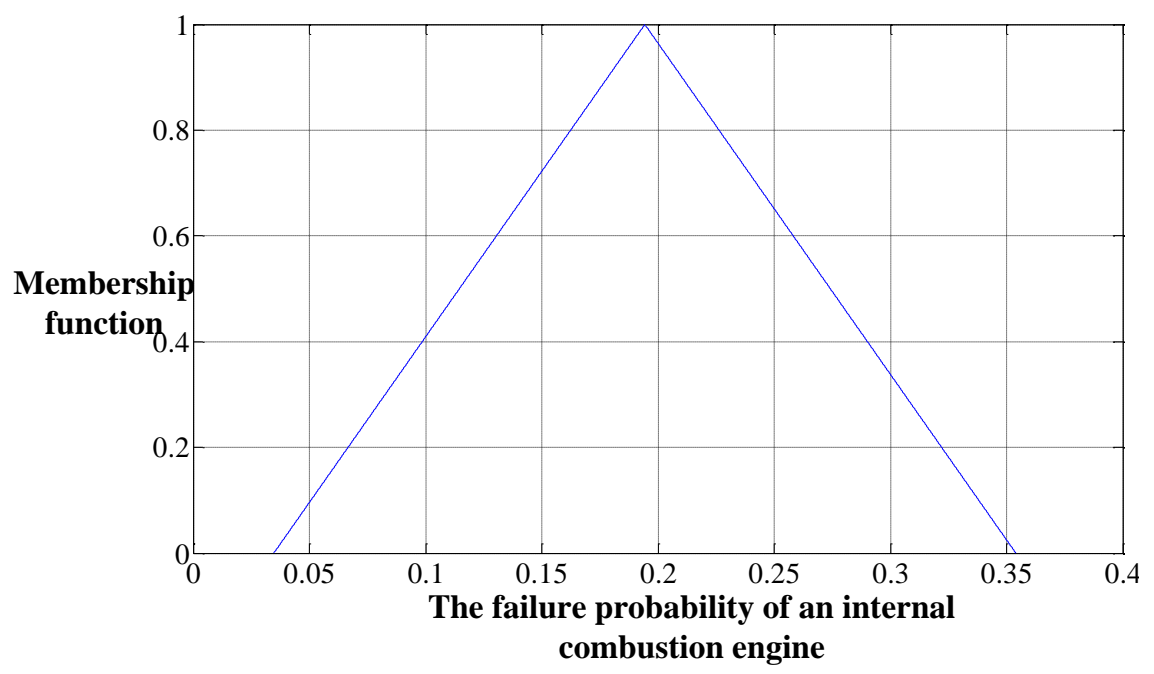

Figure-4 The membership function of the failure probability of an internal combustion engine

\section{Conclusions}

It is an important content of reliability engineering to calculate the failure probability based on the fault tree. In this paper, we establish the dynamic fault tree with Markov process, at the same time, the fuzzy uncertainty of failure of each unit is considered, which using the triangular fuzzy number and the exact probability values described at the probability of occurrence of the bottom event. Take the dynamic fault tree into a Markov process, the probability of failure dynamic fault tree can be effectively solved. Describing the fault probability of bottom events by fuzzy number theory makes full use of the historical data of each unit, and the uncertain factors of all kinds of events are relatively, then we can get the accurate failure rate estimation. The method is applied to dynamic fault tree modeling of an internal combustion engine and the calculation of fault probability to verify the effectiveness of the proposed method. The method can overcome the existing problems of traditional fault tree in the engineering application, which has a certain practical value in engineering. 


\section{References}

[1] Li Yan-feng. New method of dynamic fault tree analysis of complex system and its application [D]. University of Electronic Science and Technology of China,2013:10-28

[2] Wang Shao-ping. Engineering Reliability [M], Beijing: The publishing company of Bei Hang University, 2000:102-122.

[3] Gao Shun-chuang. Methods and Implementation of Dynamic Fault Tree Analysis [D]. National University of Defense Technology, 2015:5-33

[4] ZHU Zhengfu, LI Changfu, HE Enshan, et al. Study on System Failure Probability Model

Based on Dynamic Fault Tree[J]. Journal of China Ordnance, 2009,5(4):306-309.

[5] Cong Guangpei, Gao Jinji, Yang Jianfeng, et al. Risk Assessment Based on Fault Tree Analysis for Damaged Pipe Repair During Operation in Petrochemical Plant [J]. Transactions of Tianjin University, 2013, 19 (1): 70-78

[6] Wu Zhuang, Shi Zhu, He Xin-gui. A Fault Tree Analysis Method Based on Fuzzy Set Theory and Its Application [J].System Engineering and Electronics, 2000,22(9):72-75

[7] Yuan Long, Zhao Jiang, Li Meng. Fuzzy Fault Tree Analysis by a High Accuracy Fast Algorithm[J]. Journal of Mechanical Strength,2013,35( 6):755-759

[8] Cai Jia-kun, Song Hai-ping. Test and Analysis on solution methods to fault trees [J],Structure and Environment Engineering,2004,31(2):33-39 